\title{
Schistosomiasis presenting as acute appendicitis with mesenteric nodule filled with bilharzial ova: clinical
} report

\begin{abstract}
Schistosomiasis is a parasitic infestation in humans, commonly in developing countries. The infection manifests itself as a variety of different pathologies, based on the location of the parasite and its eggs. An unusual manifestation is that of a common surgical presentation, acute appendicitis. We present a case of a young male who underwent appendicectomy for acute appendicitis caused by a Schistosomiasis infestation, established upon pathological examination of the resected appendix.
\end{abstract}

Keywords: mesenteric thrombosis, schistosomiasis infection, diarrhoea, hematuria, normal haemoglobin, ileoceacal junction
Volume 6 Issue 4 - 2019

\author{
Amir Mounir,' Doaa M Hasan² \\ 'Head of General surgery department at Imbaba general \\ Hospital, El-Giza, Egypt \\ ${ }^{2}$ General Surgery resident at Imbaba general Hospital, El-Giza, \\ Egypt
}

Correspondence: Doaa M Hasan, General Surgery resident at Imbaba general Hospital, El-Giza, Cairo University, Egypt, Tel 02/01062838442,Email doaa_mohamad_hasan@yahoo.com

\section{Introduction}

Schistosomiasis is a tropical prevalent chronic granulomatous disease that can affect any organ. ${ }^{1}$ Clinical manifestations of Schistosomiasis vary according to schistosoma species, such as Schistosoma mansoni, Schistosoma haematobium, and Schistosoma japonicum, all of which have similar lifecycles. S. mansoni and $S$. japonicum cause gastrointestinal symptoms. Without treatment, Schistosoma typically survives in the human body for up to 5 years, but may continue up to 40 years. Chronic infection usually results in life threatening illness due to persistent tissue damage and fibrosis caused by body inflammatory reaction to eggs found in the affected organs. Ordinarily, S. mansoni infects the intestine and liver, while $S$. haematobium infects the bladder, kidney and ureters. Two uncommon exhibitions of gastrointestinal Schistosomiasis are appendicitis and chronic periodic epigastric discomfort caused by mesenteric thrombosis. ${ }^{2}$ We document here a case where patient presented with acute appendicitis due to Schistosomiasis infection, established upon histopathological investigation of the excised appendix.

\section{Clinical report}

Our case is a 28 -year-old male, who was until that time healthy. $\mathrm{He}$ came to the emergency department complaining of acute right iliac fossa pain that he had been living through for quite a few days. The pain was not associated with nausea or vomiting, and he had no noteworthy symptoms as headache, hematuria, dysuria, myalgia, arthralgia, cough, diarrhoea or rash.

Proceeding with the clinical examination, the patient had tenderness localized to the right lower quadrant of the abdomen and rebound tenderness. Lab work revealed normal hemoglobin $(14.2 \mathrm{~g} / \mathrm{L})$ and total leukocyte count $\left(22 \times 10^{9} / \mathrm{L}\right)$, no eosinophilia; platelets were $440 \times 10^{9} / \mathrm{L}$.

The preliminary diagnosis of his case was acute appendicitis. ${ }^{3}$ Pelviabdominal ultrasonography displayed nonspecific intraperitoneal inflammatory change in the area of the terminal ileum and ileoceacal junction, the patient was managed surgically and open appendicectomy was done. Upon surgical excision of the appendix, solitary nodule was found on the mesentery of the ilium about 8 inches from the ileoceacal junction (Figure 1), and excision of the nodule was done. ${ }^{4}$
Received: July 31, 2019 | Published: August |4, 2019

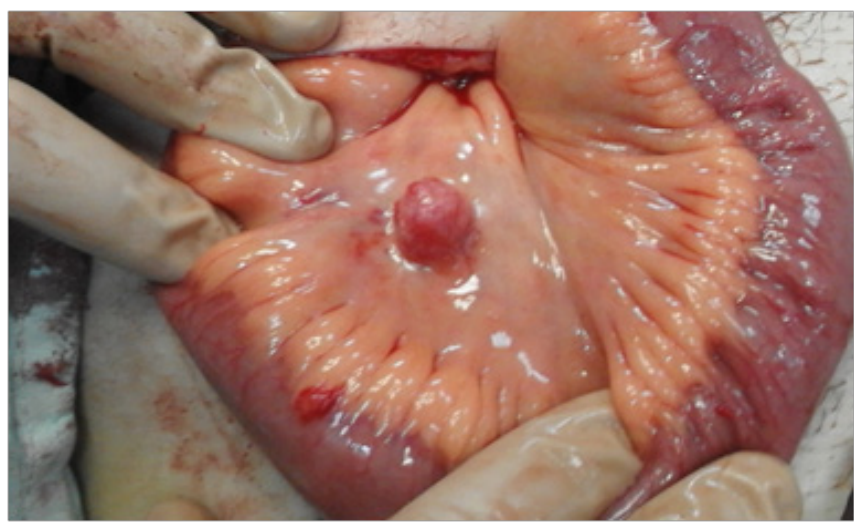

Figure I Nodule found at the mesenteric border of the ileum.

Histopathological analysis of the resected appendix and the nodule exposed acute inflammatory process, marked with copious ova found within the nodule. Further evaluation of the specimen by microbiology and parasitology lab established that these were Schistosoma ova which were designated as ovoid to spherical in shape, and with lateral spines visualized on the eggs (Figure 2). These criteria were indicative of $S$. mansoni.

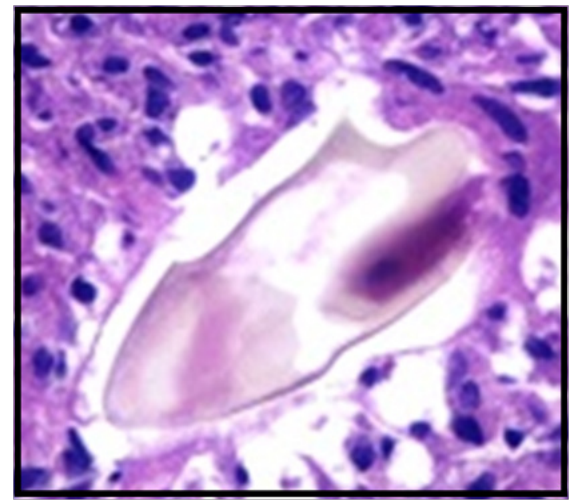

Figure 2 S. Mansoni egg inside the granulomatous nodule (H\&E [hematoxylineosin] stain) $400 x$ magnification. 
The patient was referred to the tropical disease hospital for additional assessment and treatment. Later the patient told us that he was born and had lived most of his earliest life in the rural areas of the Delta in Egypt. He was given $60 \mathrm{mg} / \mathrm{kg} / \mathrm{d}$ of praziquantel divided into 3 doses to which he was well tolerant. ${ }^{5,6}$

\section{Conclusion}

Schistosomiasis is a devastating tropical disease due to its high dominance in various developing countries. Chronic Schistosomiasis can lead to many life threatening complications, nevertheless a simple management can eliminate the parasite and stop the complication cascade. This attracts the worth of awareness of infrequent causes of common surgical presentations mostly in endemic areas like Egypt rural areas.

\section{Acknowledgments}

None.

\section{Conflicts of interest}

The authors declare there are no conflicts of interest.

\section{References}

1. Cox N, Yates P. Schistosomiasis: a rare cause of acute appendicitis. J Surg Case Reports. 2010;(4):4-4.

2. Mosli MH, Chan WW, Morava-Protzner I, et al. Schistosomiasis presenting as a case of acute appendicitis with chronic mesenteric thrombosis. Can J Infect Dis Med Microbiol. 2016:1-3.

3. Olveda DU, Li Y, Olveda RM, et al. Bilharzia: pathology, diagnosis, management and control. Trop Med Surg. 2013:1(4).

4. Aldossary M, Almabyouq F, Mashhour M, et al. Schistosomal appendicitis presenting as acute peritonitis: A case report and literature review. $J$ Heal Spec [Internet]. 2017;5(4):225.

5. Woldegerima E, Bayih AG, Tegegne Y, et al. Prevalence and reinfection rates of schistosoma mansoni and praziquantel efficacy against the parasite among primary school children in Sanja town, Northwest Ethiopia. $J$ Parasitol Res. 2019:3697216.

6. Le Govic Y, Kincaid-Smith J, Allienne JF, et al. Schistosoma haematobium - Schistosoma mansoni hybrid parasite in migrant Boy, France, 2017. Emerg Infect Dis. 2019;25(2):365-367. 